\title{
Protée
}

\section{Plasticité et signification : le cas d'Eugène Leroy}

\section{Anne Beyaert}

Volume 27, numéro 2, 1999

\section{La réception}

URI : https://id.erudit.org/iderudit/030565ar

DOI : https://doi.org/10.7202/030565ar

Aller au sommaire du numéro

Éditeur(s)

Département des arts et lettres - Université du Québec à Chicoutimi

\section{ISSN}

0300-3523 (imprimé)

1708-2307 (numérique)

Découvrir la revue

Citer cet article

Beyaert, A. (1999). Plasticité et signification : le cas d'Eugène Leroy. Protée, 27(2), 125-131. https://doi.org/10.7202/030565ar

\section{Résumé de l'article}

Si les principales théories de la sémiotique visuelle ont convenu, à côté de l'iconicité, de l'importance du plastique, il reste à trouver les moyens de rendre compte de cette dimension. Les peintures de l'artiste du nord de la France, Eugène Leroy, qui recouvre les figures jusqu'à les faire disparaître dans la matière plastique, sont un possible champ d'investigation pour envisager une prise en compte de cette dimension. Plutôt que sur les « formants » (J.-M. Floch) ou les gestalts (sémiologie topologique), l'analyse s'appuie alors sur le temps de fabrication du tableau. 


\section{PLASTICITÉ ET SIGNIFICATION : LE CAS D'EUGÈNE LEROY}

ANNE BEYAERT

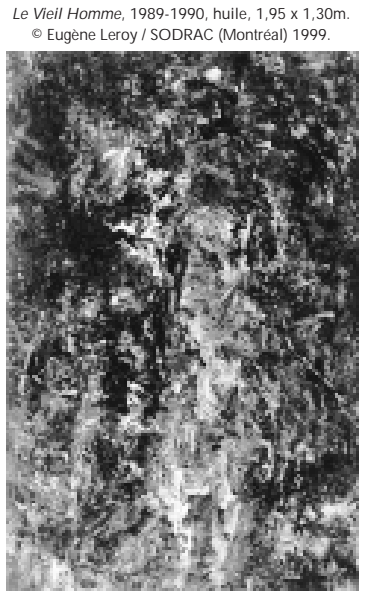

Le Groupe $\mu$ (1992: 67-68) a identifié la figure à «ce que nous soumettons à une attention impliquant un mécanisme cérébral élaboré de scrutation locale» et le fond, a contrario, à ce que nous ne soumettons pas à ce type d'attention et qui sera analysé par des mécanismes moins puissants de discrimination globale des textures. La distinction dela figureet du fond est uneinférence de la posedu contour. II suffit de tracer un contour pour conférer à une partie de I'espace un statut de figure et, à l'autre, un statut de fond. Cette distinction fondamentale fournit donc, d'emblée, un principe d'organisation de l'énoncé. $M$ ais qu'advient-il lorsque le fond «remonte» jusqu'à rejoindre la figure et jusqu'à dissoudre la différence? C'est alors la matière picturale même qui devient l'objet de l'expérience esthétique.

Cette «remontée du fond» que nous tenons pour un trait définitoire de l'haptique bouleverse les conditions de la quête du sens. Q uelles prises offre-t-elle à cette quête? Et comment se construit la signification dans l'univers du plastique? T elles sont les questions qui nous intéressent ici.

Parce qu'elles manifestent une sorte de praxis du plastique, lestoiles d'E ugène Leroy pourraient nous instruire de quelques données essentielles de cette problématique. $N$ ous étudierons plus particulièrement une toile de 19891990 peinteà l'huile, intitulée Le Vieil H ommeet mesurant $1,95 \mathrm{~m}$ sur $1,30 \mathrm{~m}^{1}$, sachant que chacune de ces caractéristiques - titre, matière et dimensions- s'avérera d'un certain intérêt heuristique pour la suite de l'étude.

Cetableau selaisse identifier à un espacetensif animéd'un mouvement dialogiqueimpliquant tout à la foisl'aspectualité, la proxémique et la configuration de la lumière. II met en tension un rapport au monde propre au style optique et à celui del'haptiqueen imprimant profondément ladynamique verticale du tableau. D ans cet espace éminemment instable où la valeur nese construit jamais quesur un fond agonistique, la quête du sens devient l'expérience d'un «être au monde».

\section{Le «monstre»}

La première rencontre d'une œuvre d'Eugène Leroy est une expérience toujours déroutante qui décourage le projet sémiotique. En l'absence deformants, au sens de J.-M . Floch, ou de gestalt si l'on préfère la terminologie de F. Saint$M$ artin, la sémiotique ne trouve guère de «prises» susceptibles de conduire l'analyse. Parce qu'elle dissout la différence, cette peinture est un monstre au sens où l'entend G. D eleuze dans D ifférence et Répétition (1989: 44):

Le fond qui remonte n'est plus au fond, mais acquiert une exi stenceautonome; la formequi seréfléchit danscefond n'est plus une forme mais une ligne abstraite agi ssant directement sur l'âme [... ] Pour produire un monstre, c'est une pauvre recette d'entasser des déterminations hétéroclites ou de surdéterminer l'animal. II vaut mieux faire monter le fond et dissoudre la forme.

La «remontée du fond» ainsi décrite s'obtient, dans la pratique, par un patient travail de recouvrement caractéristique de la production d'E. Leroy et systématisé depuis une dizained'années. Cette pratique du recouvrement offre une première prise à notre analyse parce qu'elle implique une aspectualitéet uneactantialité. $D$ ans leschéma aspectuel et actantiel de cet objet symbolique particulier qu'est le tableau, un procès de fabrication, dont le sujet-énonciateur correspond au producteur, précède un procèsd'exposition dont l'instance d'énonciation est l'observateur.

L'inscription d'une figure, celled'un vieil hommeen l'occurrence, fait office de limite inchoative au procès de fabrication. A prèsl'avoir posée, lepeintrey revient inlassablement, de jour en jour, et superpose les couches jusqu'à ce que cette figurese dissolvelittéralement dans la masse picturale, jusqu'à noyer l'iconique dans le plastique, pour ainsi dire.

Ce faisant, le producteur modifie graduellement le dispositif proxémique. II rapproche le point de vue, sachant 
néanmoinsque lepoint qui correspondait à la figureliminaire du vieil homme reste inscrit dans la mémoire du discours. Par métaphore, on évoquerait à ce propos un changement de focale semblable à celui qu'observe L. M arin (1994: 244) dans les peintures de $V$ an Eyck, qui contraint l'observateur à osciller entreune position quelque peu éloignée du tableau et maintes positions qui en sont très proches.

Lorsquel'observateur s'approche, expliqueL. M arin, «le spectacle distancié du monde se disperse, se dissout dans la prolifération des singularités, dansl'apparente fécondité des choses» (p. 245).

\section{Le lointain et le proche}

Cette méthode rappelle un procédé séculaire de la perspective déjà décrit par De V inci (1953: 37) qui, pour éloigner les objets dans la profondeur cognitive, en effaceles contours. O n objecterait néanmoins quel'effet de sens est ici plus complexe. Selon De V inci, l'effacement des contours concerne la profondeur cognitive alors que le recouvrement systématique d'E. Leroy, opérant par des touches épaisses données par des coups de brosse vigoureux, les rapproche en fait de l'observateur dans l'espace phénoménologique. En ce sens, il ne s'agirait plus de creuser l'espace mais, pour ainsi dire, de le faire avancer. Faisant dialoguer le lointain et le proche, cette sorte de perspectiveà rebours mobilise donc les deux orientations de la dynamique verticale du tableau.

C eci nous conduit à mettre en perspectivel'espacephénoménologique et la profondeur cognitive. Éloigner une figure dans la profondeur cognitive provoque toujours un éloignement concomitant du point de vue de l'observateur situé dans l'espace phénoménologique. De même, superposer les couches de peinture rapproche le point de vue tout en installant une profondeur proxémique.

II faut, pour apprécier la finesse du dispositif, emprunter à la sémiologie topologique. Celle-ci identifie les différents avatars de la perspective à autant de procédures de mise à distance La perspectiveeuclidienne, qu'elleintituleégalement creusante, installe une profondeur lointaine ou intermédiaire. À côté de ce qui s'impose comme le «canon historique» de la perspective, la sémiologie topologique reconnaît une multitude de graduations dans la distance, allant des profondeurs infinies aux profondeurs proxémiques

Envisager notreV ieil $\mathrm{H}$ ommeàl'aune de cette grammaire spatiale conduirait, sans difficulté, à lui associer une profondeur proxémi que caractéristique de la perspective optique?2. D e ce modèle, il partage la labilité, cette souplesse des plans qui caractériselestoiles deP.-É. Borduas ou, plus spécifiques, deP. M ondrian 3. La profondeur proxémiquey est, demême, constituée de plans très rapprochés les uns des autres ${ }^{4}$. Pourtant, cet espacen'en reste pas moins centré, marquéqu'il est encore par la spatialité de la perspective euclidienne. En fait, la translation figure/fond permise par l'inscription préal able du Vieil H omme continue d'organiser l'espace.

Ainsi, quoiqu'il emprunte les caractères de ce que la sémiologie topologique appelle la perspective optique, le tableau conserve le dispositif topologique de la perspective euclidienne «en mémoire». Et cet espace tensif trouve sa traduction aspectuelle dans un mouvement allant du «souvenir » du Vieil H omme conservédans son espace héritédela Renaissanceversun devenir qui, de coucheen couche, promet le tableau à la perspective optique.

\section{La strate et la couche}

Un tel mouvement aspectuel permet en tout cas d'approcher l'intimité fonctionnelle de cet objet symbolique spécifique qu'est le tableau.

Pour décrire une toile de R othko, J. Fontanille (1994: 89) initiait la catégorie strate/couche. II définissait la couche commeune surface colorée mise en place par l'artistelors du procès de fabrication, laquelle couche se distingue de la strate qui est un plan de la profondeur construit par l'observateur pour donner forme à la perspective cognitive.

Ces deux instances coucheet strate sont sous la dépendance $d^{\prime}$ un découpage actantiel et aspectuel ${ }^{5}$ qui permet derendre compte d'une conversion caractéristique de la fonction symbolique par laquelle, pour citer Panofsky, un «fairevoir dédouble conceptuellement un fairesens» 6 .

Le producteur dispose une couche qui, pour peu qu'elle soit saisie par un observateur, se convertira en strate. Elle se transformera en strate parce qu'il n'existe pas d'«œil pur», d'œil sans sujet derrière, et qu'il suffit de disposer des couleurs sur un plan pour impulser une dynamique verticale, pour que l'observateur les interprète comme des plans de profondeurs.

D onner à voir, dirait à ce propos G. Didi-H uberman, c'est toujours inquiéter le voir, dans son acte, dans son sujet. Voir, c'est toujours une opération de sujet, donc une opération refendue, inquiétée, agitée, ouverte. (1992 : 51)

La conversion symboliques'inscrit dansunequêtedu sens. La couche est une sorte d'instance basique néanmoins productrice desens et qui s'avérera signifiantepour peu qu'un 
observateur la vise ou la saisisse en y projetant un univers de valeurs ${ }^{7}$. La conversion repose sur une tension entre deux intentionnalités complémentaires qui, en transformant un unique objet, d'abord couche puis strate, participent à la production et à la saisie du sens.

Identifier la couche et la strate aux deux fonctifs de la conversion symbolique s'avère d'une certaine rentabilité. $\mathrm{C}$ ar ajouter des couches, comme le fait E. Leroy, permet certes demodifier la proxémi que pour mettre en tension lelointain et le proche, mais, du point de vue del'aspectualité, introduit aussi un délai dans le parcours du sens. Prolonger à l'envi le procès de production du tableau diffère d'autant celui d'exposition et remet à plus tard la conversion symbolique. Le travail de recouvrement introduit un retard dans la signification.

\section{La mémoire du discours}

La pratique du recouvrement trouve sa traduction dans les modes d'existence. C hacune des couches supplémentaires apposées se laisse en effet identifier à une strate potentielle que le producteur virtualisera, c'est-à-dire reléguera à un niveau de présence moindre dans l'épaisseur du discours, à moins de l'actualiser comme strate. L'actualisation déterminera d'ailleurs le statut aspectuel de la couche enfin convertie puisque celle-ci tiendra lieu de limite terminative pour le procès de production.

Pour autant, cette «coucheultime» ne produira pas le même effet de sens que si on la posait sur une toile vierge. T outes les couches conservées dans la matière picturale sont impliquées dans la conversion en strate et prendront une part qui resteà définir dans le parcours du sens. En somme, le retard aspectuel induit une «conversion globale» qui, actualisant en stratela dernière couche posée, chargera celleci de toutes les épaisseurs préalables.

Ainsi la peinture d'E. Leroy modifie-t-elle quelque peu les termes de la conversion symbolique: il ne s'agit plus de «fairesens» par l'intermédiaire du seul «faire-voir iconique», mais de faire-sens en renvoyant aussi à la matière picturale. En ce sens, la spatialité du tableau se définit par une tension entre l'iconique et le plastique, deux dimensions qui participent, par des apports gradués, à la production et àla saisie du sens.

\section{La spatialité du tableau}

Rassemblant nos différents résultats, on représentera la spatialité du Vieil H omme sur une valence, figure embléma- tique de la sémiotique tensive décrite par J. Fontanille et C. Zilberberg (1998, notamment). Les gradientsorientés qui rendent compte de l'intensité et de l'étendue selon la règle générale énoncée par ces deux auteurs sont identifiés aux dimensions du sensibleet del'intelligible, les deux fonctifs de la conversion symbolique. Une telle dénomination permet depasser du niveau d'unerègle général esusceptible derestituer tous les faits de langage, au cas particulier qu'est le système du tableau. Les deux gradients restituent les dimensions du plastique(sur l'axe du sensible) et del'iconique(l'intelligible) et leur corrélation donne forme à la spatialité du tableau. Ces directions posées, la val ence permet de représenter les deux «styles prototypiques» que sont l'optique et l'haptique par des corrélations converses où le plastique et l'iconique augmentent de façon concomitante en marquant toutefois une inflexion vers le sensible et vers l'intelligible.

D eH ildebrand à R iegl, W orringer, $M$ aldiney et $D$ eleuze en passant par H usserl, Gandelman, Aumont et Bonitzer pour le cinéma et Fontanilleà propos de la lumière-matière, la catégorie de l'haptique bénéficie d'une impressionnante généalogie. 0 n la définirait ici, par simplicité, à la façon de D eleuze comme «unepossibilitédu regard, un type de vision distinct de l'optique» (1981: 79).

Le philosophe emprunte à $\mathrm{H}$. M aldiney cette autre définition de l'haptique:

[C'est] unefaçon detâter du regard dans la mesure où, dans la zone spatiale des proches, le regard procédant comme le toucher éprouve au même lieu la présence de la forme et du fond. (1981: 195)

Sur la valence qui établit la spatialité du tableau, la peintured'E. Leroy met en tension les caractères définitoires del'optiqueet del'haptiqueet prend la formefiguratived'une tension entre les deux courbes rendant compte des deux «styles».

U ne telletension doit être envisagée dans son rapport au manuel et au visuel, qui indiquent l'origine sensible de I'information: I'iconiquesollicitela vuealors que le plastique sollicite une compétence du toucher. La différence entre un mode de perception visuel et un mode manuel peut être appréciée par une nouvelle référence à G. D eleuze (1981). Pour rendre comptedela perception appropriéeàla peinture de F rancis Bacon, le philosophe établit une hiérarchie entre le digital, le tactile et le manuel, en fonction de la plus ou moins grande subordination de la main à l'œil. D ans cette perspective, l'espace perceptif se laisse identifier à un espace 
tensif où la vue et le toucher interviennent de façon graduée. Ledigital représente al orsla moindre implication du toucher et, corrélativement, la plus grande dépendance au visuel. D ans le manuel, le rapport est inversé et la main informe seule la perception.

Se situant hors de cette hiérarchie, I'haptiques'impose, à lire G. D eleuze, comme une compétence particulière de la vue:

On parlera d'haptique chaque fois qu'il n'y aura plus subordination étroite dans un sens ou dans l'autre, ni la subordination relâchée ou connexion virtuelle, mais quand la vuedécouvrira ains unefonction detoucher qui lui et propre, et n'appartient qu'à elle, distincte de sa fonction optique. On dirait alors que le peintre peint avec ses yeux, mais seulement en tant qu'il touche avec ses yeux. (p. 99)

Représenter l'optique et I'haptique sous la forme d'une corrélation converse dont la courbe s'infléchit en direction du sensible (l'haptique) et de l'intelligible (l'optique) nous amène logiquement à nous interroger sur la nature du point d'inflexion que R. Thom appellerait point critique, où s'effectuela discontinuitésignificative. $N$ otrehypothèse est que ce point critique correspond à cet état de l'aspectualité où le fond remonte à la surface jusqu'à «invalider» la distinction figurelfond, jusqu'à dissoudre la différence dirait G. D eleuze.

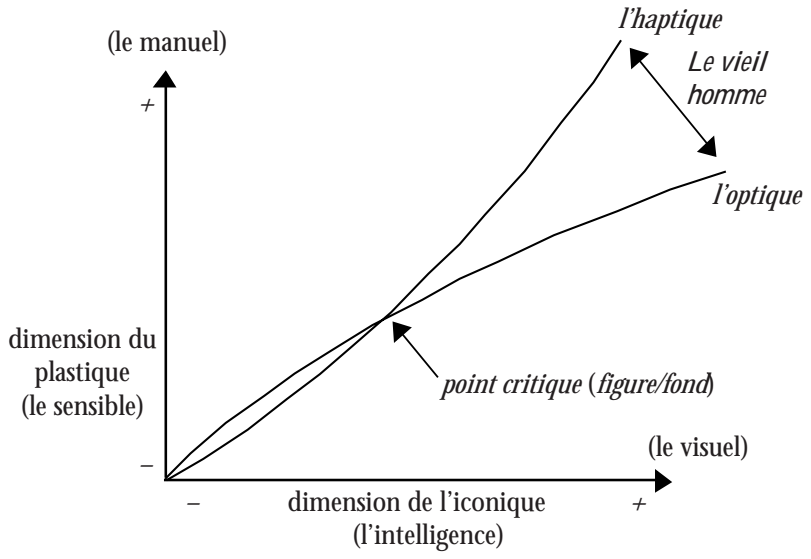

La valence de la spatialité du Vieil H omme d'E. Leroy

\section{La quête du sens}

Le peintre a intitulé le tableau Le Vieil H omme, mettant d'emblée son observateur dans la situation d'un sujet de quête qui tâchera d'extraire la figure éponyme des profondeurs du tableau. La quête du sens s'appuie dès lors sur le dispositif chromatique.
Le brun-rouge est une base permanente dans la peinture de Leroy. Les enfants I'obtiennent en mélangeant toutes les couleurs de leurs boîtes de gouaches et il est donc tout à fait prévisible qu'un travail de recouvrement la fasse émerger. $C$ ette couleur s'obtient plus simplement en mêlant du rouge et du vert, ce qui dans la pratique revient au même puisque les trois couleurs primaires sont al ors associées, mais s'avère d'unecertainerentabilitéd'un point devueépistémologique puisque ce nouveau mélange nous instruit de la position du plan dans la profondeur cognitive.

Retenant le trait du chromatisme pour insérer le brunrouge dans la perspectiveatmosphérique, l'observateur l'interprétera en effet comme un plan s'inscrivant dans une profondeur restreinte, à mi-chemin entre le rouge, compris comme un plan rapproché, et le vert, compris comme un plan intermédiaire.

S'agissant de la tonal itéé, I'observateur identifiera ce plan à un complexe clair-sombre tendant vers le sombre, soit de même comme une profondeur restreinte dans laquelle les touches claires susceptibles d'évoquer le Vieil Homme creusent des plans plus ou moins éloignés.

$M$ aisl'analyse pourrait être affinée. À vrai dire, la couleur brun-rouge s'approche davantage du rouge que du vert et sera donc perçue comme un plan plutôt avancé. Q uand aux touches bleues, jaunes, vertes, rouges qui animent cet espace proxémique, elles prennent placeà des niveaux deprofondeur extrêmement variables allant du très proche au plus lointain, mais tendant toujours à s'approcher. Cette frange proche et labileest circonscritepar deux limites aspectuelles: une limite proche donnée par le rouge (chromatisme) et le brun-noir (tonalité), et une limite lointaine correspondant au bleu (chromatisme) et surtout au blanc (tonalité).

La matrice brun-rouge s'impose de ce fait comme un plan intermédiairebalayéd'un mouvement dialogique allant du très proche au plus éloigné, entre les plans extrêmes que forment, pour la tonalité, le sombre de la périphérie et le clair des parties centrales et, pour le chromatisme, les touches rouges et bleues.

\section{La quête de la lumière}

La complexité du dispositif permet, de surcroît, de connoter le parcours de quête del'observateur d'une val eur lumineuse. Rechercher le Vieil H omme du titre dans la matière colorée revient en fait à reconstruire la configuration de la lumière, de sortequel'observateur prend acte, dans sa quête 
du sens, d'unetension entre deux conceptions dela perspective atmosphérique.

La couleur matricielle est, en effet, le résultat d'un travail de mélange des pâtes. En se superposant, les couleurs se modifient pour aboutir à cette sorte determe complexe qu'est le brun-rouge. Cependant, des couleurs ont «échappé» au mélange et restent identifiables, sansqu'il soit dit néanmoins qu'une nouvelle couche ne ferait pas «surnager» le brunrouge matriciel.

Ainsi le travail de recouvrement induit-il deux façons d'obtenir la couleur: I'unes'effectue par mélange despigments et aboutit au brun-rouge; l'autre permet de conserver d'autres taches colorées préservées pour ainsi dire d'une disparition danslebrun-rouge. M ais préservent-elles vraiment du brunrouge?C ette seconde manière doit, àvrai dire, être considérée commeune autrefaçon d'y aboutir en procédant par mélange optique.

Ce procédé systématisé au XIX ${ }^{\mathrm{e}}$ siècle et qu'illustre la peinture de Seurat est défini notamment par $F$. Saint-M artin (1987: 64). Le mélange optique veut que deux couleurs juxtaposées, et perçues à une certaine distance, produisent dans l'œil une troisième couleur appelée couleur résultante. L'action des deux couleurs initiales se trouve en quelque sorte annulée, rendue imperceptible au profit de la troisième couleur. En ce sens, le mélange optique réunissant toutes les couleurs de la palette s'impose comme une autre façon d'obtenir du brun-rouge, sous forme de couleur résultante. Un tel mélange a pour conséquence de reconsidérer la proxémi que puisque, pour apprécier cette couleur résultante, l'observateur est contraint de prendre ses distances, de s'éloigner de l'énoncé.

F. Saint-M artin précise en outre qu'au contraire d'une couleur résultant du mélange des pigments, celle obtenue par mélange optique conserve toute sa luminosité. Si I'on conviendra d'une capacité particulière du mélange optiqueà garantir ce que cet auteur appelle la lumi nosité, soit à activer cequeJ. Fontanille (1995) désignerait commel'état dechromatisme de la lumière, on soutiendra que le brun-rouge obtenu par mélange de pigments mobilise d'autres états de la lumière. Car ce brun-rouge matriciel n'a pas été obtenu sur la palette du peintre mais sur le tableau même. En cela, le procédé de fabrication d'E. Leroy déroge à la canonique méthode additive pour se «colleter à la matière» en quelque sorte. Et cette matière se présente comme une texture extrêmement instable qui engendre des effets de sens bien diffé rents de ceux que produirait la surface colorée d'une toile vierge, marquée par lerythme régulier du tissage. Elle renvoie à différentes sortes de texture, à différentes propriétés de la masse coloréequi, selon l'inclinaison des couches, réfléchiront ou absorberont différemment les rayons lumineux. Pour parler commeR. Thom, cette textureen perpétuelle agitation opposera diverses discontinuités qual itatives à la propagation de la lumière. Elle activera, dirait quant à lui J. Fontanille (1995), différents effets de lumière matière, d'édat, voiredes modifications de la couleur.

Ainsi la quête du sens selaisse-t-elleidentifier à unequête dela lumièrequi aboutira à séparer deux versions de la couleur matricielle induisant des dispositifs proxémiques différents. $M$ ais séparer n'est pas encore le mot qui convient: la quête du sens induit une discrimination qui permet de distinguer une configuration de la lumière, propre au Vieil Homme, d'une autre configuration. En somme, il ne s'agit plus de distinguer une figure d'un fond, mais la lumière d'une figure de la lumière du fond.

\section{Un «être au monde»}

II va de soi que les compétences requises du sujet percevant diffèrent selon quela dimension du plastiqueet de l'iconique est plus ou moins prise en compte. L'iconique requiert une compétence visuelle et identifie le sujet à un corps-position qui saura apprécier les distances et convertir la couleur en profondeur. Le plastique sous-tend une compétence manuelle; il mobilise un corps-chair, un corps sensori-moteur qui perçoit par la main. En ce sens, on formerait I'hypothèsequeletableau d'E. Leroy sollicite deux instances del'énonciation: un sujet-observateur et cettetoute première instance du discours qu'est la «chair » phénoménologique. La compétence cognitive de l'observateur, acquise au contact du visible du style optique, déboucherait ainsi sur une autrecompétence du sujet, amenéà «ressentir», de sortequel'existence sémiotique du sujet serait, elle aussi, affectée par la mise en perspective de l'optique et de l'haptique, du lointain et du proche, du «souvenir » et du devenir.

À ce sujet sollicité à la fois comme observateur et comme «chair» phénoménologique est proposée une expérience synesthésique. D ansl'œuvre pictural e tout s'adresse à la vue mais tout n'est pas visuel, et la synesthésies'impose ici comme une autrefaçon de voir. Explorant le concept de synesthésie, H . Parret (1995) a montréqueles cinq sens- la vue, l'odorat, le goût, le toucher et l'ouïe- communiquent synesthésiquement sur le fond d'un toucher fondamental qui constitue I'origine commune, dite pré esthésique, des cinq sens. À lire 
cet auteur, la synesthésie ne relèverait donc pas d'une perception osmotique, mais d'une instance particulière de la perception régie par ce toucher fondamental.

Pour étayer la thèse d'une expérience synesthésique s'appuyant sur un toucher fondamental mobilisant les cinq sens, on évoquerait certes cette capacité particulière de l'haptique à «donner des mains au regard». Les innombrables couches de peinture accumulées répandent, en outre, dans I'air de fortes odeurs d'huile de lin et de térébenthine qui sollicitent l'odorat et, pour peu que plusieurs tableaux soient rassemblés, incommodent passablement I'observateur ${ }^{9}$. Parmi les cinq sens reconnus par la tradition, le toucher synesthésique fondamental mobiliserait donc essentiellement la vue, le toucher et I'odorat.

U nepossibilitéserait encore, pour reconstruirecetoucher fondamental, d'associer aux cinq sens reconnus par la tradition philosophique des dimensions de la perception aperçues par les sciences cognitives. Ainsi évoquerait-on la dimension du postural qui joue dans la relation de proportion entrelecorps propre et le tableau (F. Saint-M artin, 1987: 192-193). Le format vertical du tableau et ses proportionsévoquent la station debout d'un être humain. Ses dimensions dépassent légèrement la taillenormalisée de cet homme- la grandeur naturecomme s'il invitait cette instance à la confrontation.

La tactilité caractéristique de l'haptique pourrait encore recevoir une connotation kinesthésique, une dimension qui rend compte du trajet physique dans la perception et de la possibilité de préhension et de manipulation. En dépit de ses grandes dimensions, le format du tableau permet de surcroît une manipulation et un transport assez aisés 10 .

L'organisation de la profondeur oblige en outre l'observateur à se déplacer pour osciller entre des points de vue plus ou moins proches. Ainsi le vieil homme rejoindrait-il ces œuvres $d u x x^{e}$ siècle où J. Lupien, tirant profit des résultats dessciences cognitives, reconnaît des espaces tactiles kinesthésques.

II importe d'ailleurs de souligner l'importance de la dimension synesthésique ainsi augmentée de composantes posturales et kinesthésiques pour la quête du sens. À vrai dire, si le cartel ne l'en instruisait pas, l'observateur aurait bien peu de chances d'identifier un vieil homme. Toutefois, le format vertical du tableau, ses dimensions et la position qu'y prend la «forme lumineuse» lui indiqueraient à l'évidence que cette forme lumineuse qui apparaît face à lui est sans conteste un être humain, qu'on spécifierait même comme adulte, un être humain qui avance vers son interlo- cuteur, tous deux se cherchant dans leur profondeur instable.

Le vieil homme propose une expérience synesthésique, où la valeur seconstruit sur fond agonistique par le dialogue de l'optique et de l'haptique, de l'iconique et du plastique, requérant du sujet une compétence visuelle autant que manuelle et le considérant à la foiscommeun sujet-observateur et comme une simple «chair» phénoménologique.

$H$. Parret définit encore la synesthésie comme une expérience de «l'unitédesoi, dela chosemêmeet du monde» 11 . Elle donne lieu à une expérience intime, ipséque pourraiton dire, par laquelle le sujet se définit dans un rapport à l'autre et au monde. U ne telle expérience ipsé que passe ici par la confrontation de deux chairs, la «chair » métaphorique de la peinture, qui se confond avec celle du vieil homme portraituré, et la «chair» phénoménologique du sujet percevant. Et la quête du sens s'inscrit dans une tension où l'expérience de l'une passe par la «conquête» de l'autre.

\section{Conclusion}

Cherchant les «prises» qui permettraient de rendre compte de la dimension plastique du «vieil homme», nous nous sommes appuyée sur une aspectualité. C e fai sant, nous nous sommes intéressée au procès de fabrication du tableau, dérogeant ainsi aux habitudes sémiotiques qui veulent que I'analyste adopte le point de vue de l'observateur. Se référer au plastique implique donc que le temps correspondant à la fabrication du tableau soit pris en compte, ce qui permet de faire de la couche une instance susceptible de signifier également, «hormis» sa conversion en strate. $D$ ans sa quête du sens, l'observateur convertit certes la «couche ultime» en strate, mais toutes les couches antérieures entrent aussi en ligne de compte pour en «charger» le sémantismeet opposer un dispositif complexe à la propagation de la lumière.

En ce sens, l'aspectualité du plastique induit une prise en compte du temps. Elle implique même que le temps soit rabattu sur la profondeur et qu'il instruise donc la proxé mique. Ce faisant, I'aspectualité confronte deux représentations du temps, le temps de la fabrication et le temps de I'observation, qu'elleposetous deux comme porteurs de sens.

La prise en compte del'aspectualité, de ce temps rabattu sur la profondeur, amèneà considérer la notion de gestualité. Elle révèle que le tableau se comprend comme un objet symbolique spécifique dont la spécificité est dictée, en première instance, par une sensori-motricité particulière. $\mathrm{N}$ otre Vieil Homme n'est pas proposé par une fresque, un volume ou un vase, ces autres objets symboliques qui im- 
pliquent une sensori-motricité toujours différente. Le parcours du sens qui mène à lui est entièrement déterminé et instruit en chemin par une sensori-motricité propre au tableau.

Tâchant de définir cettesensori-motricitédéfinitoire, on formeraitl'hypothèsed'une sortedesensori-motricitéglobale qui concernerait les deux instancesénonçantes, leproducteur qui est aussi le premier observateur du tableau, et l'observateur proprement dit. Selon qu'il s'agit d'un tableau, d'unefresque, d'un volume ou d'un vase, une «ressemblance» entre les deux gestuelles apparaît: danslevolume, les deux instances doivent «tourner autour »commeleprescrit l'étymon volumen ; le vase sous-entend une manipulation; la fresque, la déambulation le long d'une architecture... En ce sens, la sensori-motricité qui anime chacun de ces objets symboliques procède par une sorte d'échoïsation du geste qui reproduit le schéma aspectuel et actantiel du tableau : à la gestuelle du producteur répond celle de l'observateur. Et cette gestuelle «en miroir» détermine les dimensions posturale et kinesthésique de chaque objet symbolique, soit les questions des proportions et du trajet physique, avant toutes les autres.

La prise en compte du temps de la fabrication et d'une sensori-motricité spécifique au tableau s'impose en tout cas comme un viatique stimulant permettant d'avancer dans la compréhension du plastique, cette dimension dont tous les univers théoriques, de la sémiotique de J.-M. Floch12 à la sémiologie topologique (voir notamment Saint-M artin, 1987) s'accordent à reconnaître l'importance tout en cherchant les «prises» qui permettront l'analyse.

\section{N OTES}

1. C ette toile était présentée lors de la D okumenta IX de Kassel, en 1992. Elle est reproduite à la page 331 du catalogue.

2. La perspective optique telle qu'elle est définie par l'École québécoise est sans rapport avec ce que nous définirons comme le prototype optique.

3. Lestoiles dePiet M ondrian relèvent plus précisément d'une perspective en damier ou d'une grille moderniste, voir F. Saint-M artin (1987: 168 et 172).

4. Ce caractère général doit néanmoins être relativisé. F. Saint-M artin (1987: 168) et M . C arani (1991) ont observé dans des toiles du Q uébécois P. E. Borduas, juxtaposant desplansnoirset desplansblancs, uneconversion, une équivalence del'indéfiniment lointain et del'indéfiniment rapproché 5. À ce découpage, on objecterait que le producteur peut, lui aussi, être assimilé à un observateur. U ne telle objection prendrait acte du fait que la fonction communicative du langage, quelle que soit la forme de ce langage, peut s'exercer au sein d'un même individu qui est à la fois destinateur et destinatairedu message qu'il élabore. 0 n soutiendrait néanmoins que notre découpageaspectuel et actantiel répond àun souci herméneutiqueautant que méthodologique. L'aspectualitédu tableau est orientée par unelogiquedela réception. I dentifier letableau àun objet symboliquesous-entend lacoprésence de deux instances, un producteur et un observateur, où l'un soumet à l'autre une représentation de son propre rapport au monde pour aboutir à une confrontation des deux points de vue.

6. La citation est tirée de M . C arani (1997) qui recherche chez cet auteur des fondements présémiotiques de l'histoire de l'art.

7. L'intentionnalitédu producteur consisteà servir la quêtedu sens sachant toutefoisquesa compétencenel'autorisera pasàsesoustraireàcetteexigence du sens. Par conséquent, le producteur assume une structure modale correspondant à un /ne pas pouvoir ne pas fairel qui, en l'occurrence, ne renvoie pas à une quel conque val eur modale d'obés ssance comme le suggère la règle générale définie par G reimas et Courtés (1993: 286-287), mais se fonde sur une impossibilité pour ainsi dire matérielle d'échapper à la compétence cognitive de l'observateur.

8. Lavaleur pour les plasticiens.

9. L'accrochage des toiles d'E. Leroy, lors de la Dokumenta de 1992, manifestait pleinement cette dimension olfactive, quasiment algique. Elles étaient présentées lesunes contreles autres dansun lieu exigu, situéàl'étage, extrêmement fréquenté, et contraignaient quasiment le visiteur au contact physique avec elles et avec les autres visiteurs. Une odeur extrêmement incommodante obligeait en outre le visiteur, proche du malaise, à circuler rapidement.

10. $D$ ans son atelier, le peintre adore d'ailleurs manipuler ses tableaux, les superposer lesuns sur les autres, inlassablement, commes'il s'agissait encore de couches ainsi que le montrent les photos de $M$ arina Bourdoncle.

11. $N$ ous nous référons également à une intervention faite à Paris dans le cadre du séminaire du CN RS/EH ESS, le 1er avril 1998.

12. LesPetitesM ythologiesdel'œil et del'esprit (1985) étaient déjà sous-titrées Pour une sémiotique plastique.

\section{RÉFÉREN CES BIBLIO GRAPHIQUES}

CARAN I, M . [1997]: «D e Shapiro à Panofsky, et après», Proceeding of the Fifth Congress of the international association for semiotic studies, Berkeley, Berlin/N ew York, M outon de G ruyter.

DeLEUZE, Gilles [1981] : Francis Bacon, logique de la sensation, Paris, La Différence;

[1989]: D ifférence et Répétition, Paris, P.U.F.

DIDI-H U BeRM AN , G . [1992]: Cequenousvoyons, cequi nousregarde, Paris, Minuit.

FLOCH , J.-M . [1985] : Petites M ythologies del'œil et del'esprit, Paris, H adèsBenjamins.

FON TAN ILLE, J. [1995] : Sémi otiquedu visible, D esmondesdelumière, Paris, P.U.F.

FON TAN ILLE, J. [1994]: «Sans titre... ou sans contenu », dans N ouveaux actes sémi otiques, nos 34-35-36: A pproches sémi otiques sur Rothko, Limoges, PULIM.

Fon TAN ILle, J. et ZILberberg, C. [1998] : Tension et Signification, Liège, $M$ ardaga.

GREIM AS, A.-J. et]. C C U RTÉS [1993] : D ictionnaireraisonnédela théoriedu langage, tome 1 , Paris, $\mathrm{H}$ achette.

G roupe $\mu$ [1992]: T raité du signe visue, Paris, Le Seuil.

LUPIEN , ]. [1991] : «Perception polysen sorielleet langage pictural », D egrés, n 67, Recherches québécoises, el-el7.

Panofsky, E. [1975] : La perspective commeforme symbolique, Paris, M inuit. M ARIN , L. [1994]: D ela représentation, Paris, G allimard-Le Seuil.

PARRET, H. [1995] : «Synesthesic effects», Advancesin visual semiotics, The semiotic web, 1992-93, T. A. Sebeok et J. U miker-Sebeok (édit.), M outon de G ruyter, Berlin/N ew York, 335-347.

SAIN T-M ARTIN , F. [1987]: Sémiologie du langage visuel, Sillery, Presses de I'U niversité de Q uébec;

[1991]: «Fondements sémantiques des grammaires spatiales», D egrés, n 67, Recherches québécoises, b1-b23.

VINCI, L. de [1953] : "C arnets», dans Bibliothèque mondiale, no 19, Paris. 